\title{
Recent Changes in Instructions for Use for the Amplatzer Atrial Septal Defect Occluder: How to Incorporate These Changes While Using Transesophageal Echocardiography or Intracardiac Echocardiography?
}

\author{
Kiran Mallula $\cdot$ Zahid Amin
}

Received: 6 April 2012/ Accepted: 13 April 2012/Published online: 11 May 2012

(C) Springer Science+Business Media, LLC 2012

\begin{abstract}
The Amplatzer septal occluder is commonly used to close secundum atrial septal defects (ASDs). Recently, the manufacturer's recommendations have been modified with regard to contraindications for its use. These changes primarily focus on evaluation of the aortic rim (anterio-superior rim) with transesophageal echocardiography (TEE) and intracardiac echocardiography (ICE). In this editorial, we will review systematically all of the sequential views in TEE and ICE that are required for thorough assessment of the aortic rim and provide explanation of what constitutes deficiency of the aortic rim as seen on ICE.
\end{abstract}

Keywords Secundum atrial septal defect · Intracardiac echocardiography · Transesophageal echocardiography

Secundum ASD is a defect in the septum primum that results in a communication between left and right atria. The size of the defect is primarily based on the extent of septum primum deficiency. The septum secundum is always present in these patients and constitutes the atrial septal rims, although it could be deficient in some areas. When deficient, the defect can be very large and technically difficult to close. The secundum atrial septal defect (ASD) is surrounded by the aortic, superior, superior vena cava (SVC), posterior, inferior vena cava (IVC), and atrioventricular (AV) valve rims [1]. In general, excellent short-, mid-, and even late-term results have been reported with the

K. Mallula $\cdot$ Z. Amin $(\bowtie)$

Rush Center for Congenital and Structural Heart Disease,

Rush University Medical Center, 1653 W. Harrison Street,

Jones 770, Chicago, IL 60612, USA

e-mail: zahid_amin@rush.edu percutaneous closure of ASD using the Amplatzer septal occluder (ASO) and other devices [10, 12]. However, the procedure carries a risk of early and late complications, including thrombus formation, device embolization, wire fracture, nickel allergy, arrhythmias, cerebrovascular accident, and atrial wall with occasional aortic wall erosion. The worst of these complications is atrial wall erosion, also called "perforation" $[2,6,7]$. Based on the description of surgeons, the erosions occur in the anteriorsuperior region of the atrial wall, an area that corresponds to the aortic and the superior rim on echocardiography. Although the primary reason for erosion has been attributed to device over-sizing, there have been cases in which there was no apparent device oversizing, however device placement did result in erosion [8].

More than $40 \%$ of patients with ASD have an aortic rim $<5 \mathrm{~mm}$ [11]. In a recent review of cases of erosions by St. Jude Medical (SJM), aortic rim deficiency was present in $88 \%$ of the cases in which erosion occurred. This number is consistent with our previous study [2]. Based on this analysis, aortic rim absence was a strong predictor of erosion, and so SJM updated the instructions for use (IFU) in January 2012 [4]. The changes included contraindications to close ASD with echocardiographic evidence of absent or deficient anterior-superior rim (sufficient rim was defined as the presence of at least $5 \mathrm{~mm}$ of rim in multiple and sequential short-axis views as confirmed by ICE or TEE).

In the original IFU, a $5 \mathrm{~mm}$ minimum rim was required for optimal closure of ASD for all rims except the aortic rim. The $5 \mathrm{~mm}$ sufficiency of atrial septal rims was based on the fact that the right atrial disc size is $5 \mathrm{~mm}$ larger than the waist for all sizes of devices ranging from 11 to $40 \mathrm{~mm}$. The aortic rim was not included because the device sat astride the aorta (Ao) in cases of aortic rim deficiency, and the Ao became a part of the aortic rim; thus, no cases of 
erosions occurred or were reported until the device was approved by the US Food and Drug Administration in December 2001. The recently revised IFU has had all interventionalists wondering how to adopt and incorporate these changes into their practice to ensure compliance with revised IFU because the aortic rim deficiency became a contraindication to ASO placement.

In this report, we intend to revisit the subject of ASD rims, slightly modify our original classification, define sequential interrogation of the aortic and superior rims by TEE, and describe some ICE views that would encompass or mimic sequential views only possible with TEE.

\section{Modification to the Original Classification of Atrial Septal Rims}

In our previous description [1], we described six atrial septal rims based on the adjacent structures of the heart surrounding the rims. For example, the rim toward the SVC was named the "SVC rim"; the rim toward the AV valve was named the "AV valve rim" and so forth (Fig. 1). TEE evaluates all six atrial septal rims in three views: short-axis aortic view for the aortic and posterior rims, bicaval view for the SVC and IVC rims; and four-chamber view for the $\mathrm{AV}$ valves and superior rim. We have renamed the superior rim toward the right upper pulmonary vein (RUPV) the "RUPV rim" because true superior rim by TEE cannot be seen easily, and, for the sake of simplicity, we had labeled the RUPV rim as the superior rim in our original classification. We have incorporated the true superior rim into the aortic rim and will refer to it as the "aortic rim" in this article (Fig. 2) unless stated otherwise. The description of the remaining atrial septal rims will remain the same as described previously.

\section{TEE}

TEE has been the "gold standard" for ASD closure since its first description [9]. To effectively interrogate ASD anatomy, the ASD is usually evaluated in three standard views: the four-chamber view for the AV valve rim and the RUPV rim (new classification); the short-axis view for the aortic and the posterior rims; and the bicaval view for the SVC and the IVC rims. In general, the four-chamber view is seen at $0^{\circ}$ (Fig. 3a, b). The angles range from $0^{\circ}$ to approximately $20^{\circ}$. This view can demonstrate the coronary sinus (CS) posteriorly and the most posterior part of the Ao when angled anteriorly. Hence, this view is excellent in ascertaining the adequacy of the CS rim when closing the secundum ASD. It also helps to interrogate the portion of the atrial septal rim where the Ao starts to appear in the

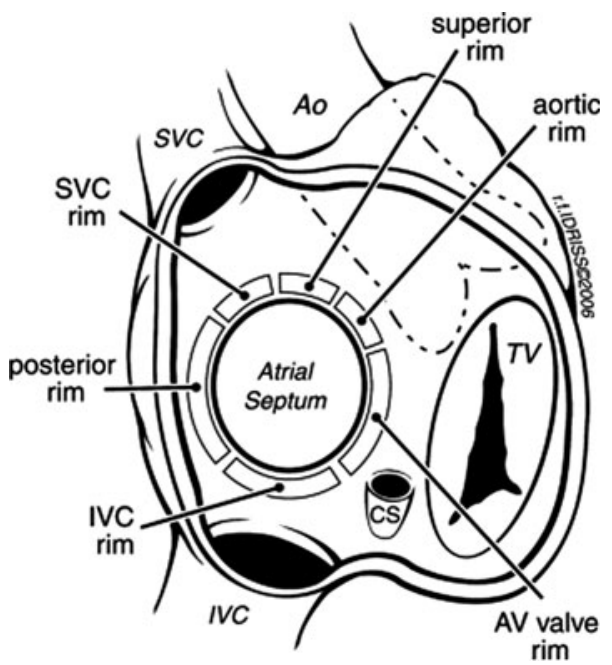

Fig. 1 Classification of atrial rims. Reproduced with permission from Catheterization and Cardiovascular Interventions. 2006;68:778-787

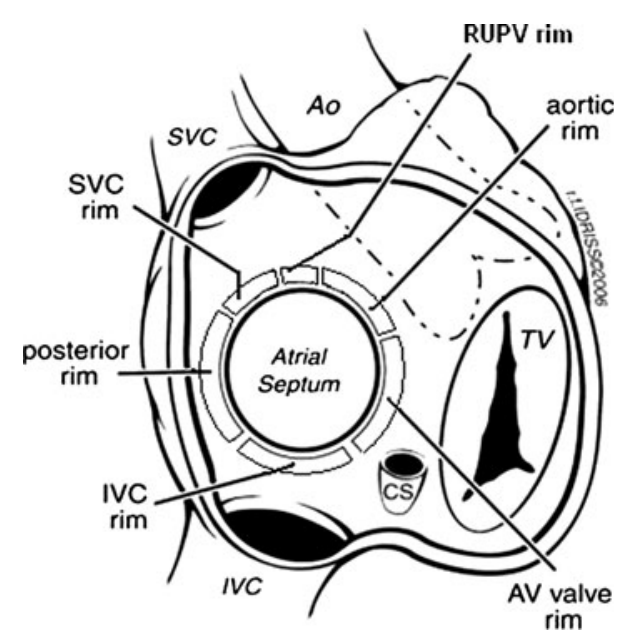

Fig. 2 New modified classification of atrial rims. SVC (Superior Vena Cava), RUPV (Right upper pulmonary vein), IVC (Inferior vena cava), CS (coronary sinus), AV (atrio-ventricular), Ao (Aorta)

image. The short-axis view spans from approximately $\geq 20^{\circ}$ to approximately $\geq 80^{\circ}$ (Fig. $4 \mathrm{a}-\mathrm{g}$ ). This view is ideal for interrogation of the aortic rim and continues to the origin of the RUPV rim. The bicaval view ranges from approximately $\geq 80$ to $\geq 120^{\circ}$. It demonstrates the $\mathrm{SVC}$ rim around $120^{\circ}$. For IVC rim evaluation, the transducer may have to be pushed in and the angles changed to lower ones, such as $90^{\circ}$.

\section{Updated Recommendations per Changes in the IFU}

We recently published the interrogation scheme to effectively interrogate the aortic rim [5]. For complete evaluation, we recommended that the aortic rim be viewed at multiple levels to adequately assess the extent of aortic rim 


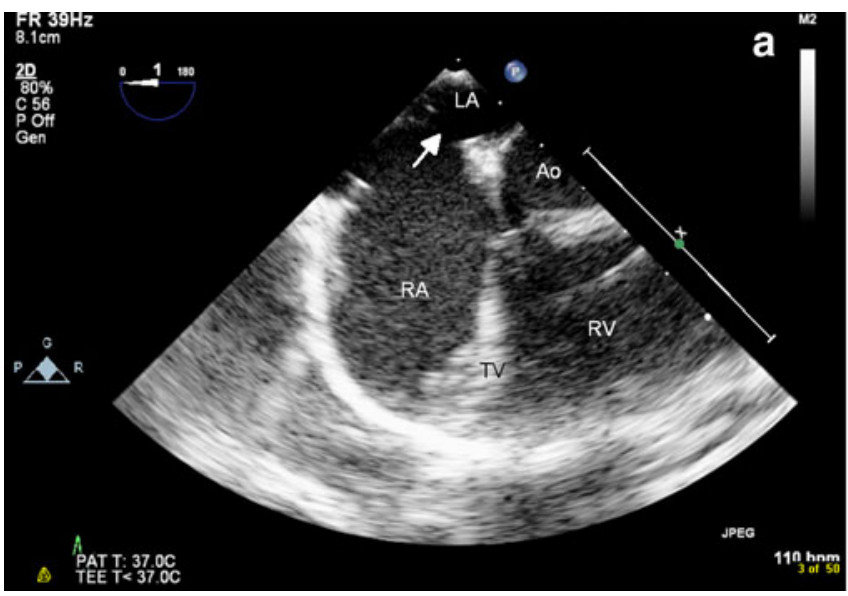

Fig. 3 (a) Four-chamber view in TEE shows the left atrium (LA), right atrium (RA), aortic sinus (Ao), tricuspid valve (TV), and right ventricle (RV) anteriorly. (b) Four-chamber view in TEE shows the

deficiency. Aortic rim deficiency or absence in one view does not preclude device placement. However, if the aortic rim is absent in three or more consecutive views, it means that the extent of its deficiency is significant and hence there may be high risk for device closure. This is precisely what was recommended by SJM in the updated IFU.

During intraprocedural TEE, the aortic rim should be measured at 20,30, 40, 50,60, 70, and $80^{\circ}$ (Fig. 4). If the aortic rim is deficient in multiple (three or more) consecutive views, the patient may not be a good candidate for device placement. However, if it was deficient in only one or two views, the patient is not a high-risk candidate for device closure with proper size selection of the device; thus, device placement is not a contraindication.

\section{Interrogation of the Aortic Rim by ICE: What Do Multiple and Sequential Views Imply?}

The adequacy of ICE while performing ASD closure has been well documented [3]. ICE can effectively assess the adequacy of SVC and IVC rims in the bicaval view and the aortic and the posterior rims in the short-axis view. There is, however, no true four-chamber view because the ICE catheter sits in the right atrium (RA). The AV valve rim can be viewed effectively with ICE. The adequacy of the $\mathrm{AV}$ valve rim can be evaluated while performing the sweep in neutral position with clock-wise rotation of the ICE catheter. The true superior rim (superior part of the aortic rim per the new classification) can be interrogated as well and is described later in detail.

For users of ICE, it is fairly obvious that no ICE views are available to interrogate the aortic rim in multiple and sequential views that would correlate with TEE views. However, we do believe that different parts of the aortic

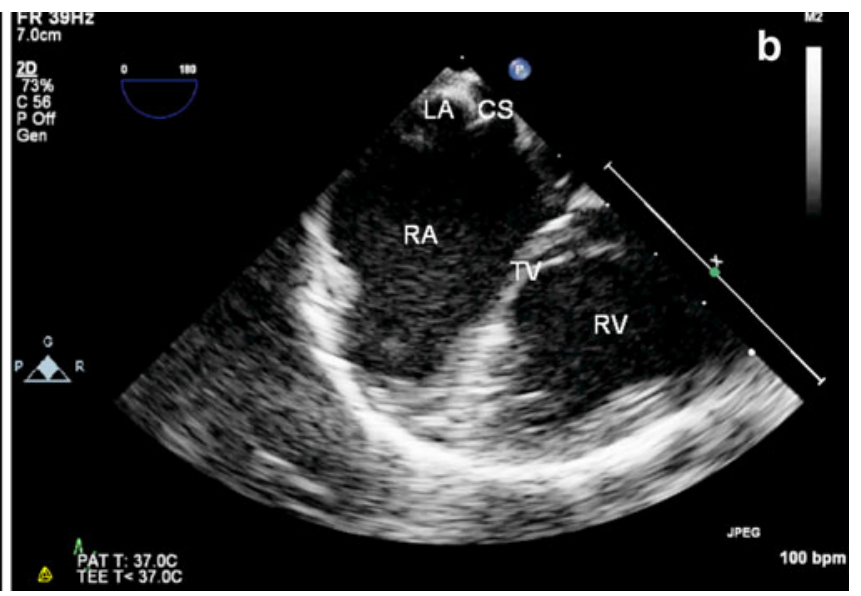

coronary sinus (CS), right atrium (RA), left atrium (LA) with ASD, tricuspid valve (TV), and right ventricle (RV)

rim can be interrogated and can help the physician to ascertain whether ASD closure is high risk or otherwise.

Here is the proposed scheme for aortic rim interrogation. A typical starting location is the home view or neutral view (Fig. 5). In this view, the RA, tricuspid valve (TV), right ventricle (RV), right-ventricular outflow tract (RVOT), pulmonary valve, proximal main pulmonary artery, a portion of the aortic valve (AoV), and the ASD with adjacent septum in partial short-axis view can be seen. Hence, this view visualizes the lower-most portion of the atrial septal rim that is analogous to four-chamber view in TEE where the Ao starts emerging $\left(\geq 20^{\circ}\right.$ if the TEE transducer is pulled back slightly).

From the home view, the anterior-posterior knob is rotated posteriorly and the right-left knob rightward to obtain the atrial view, also known as the septal view. This will enable the transducer to face the atrial septum. In this view, the entire length of the atrial septum, RA, left atrium (LA), and, occasionally, the right pulmonary venous return to the LA and CS can be seen. The terms "septal view" and "atrial view" are misnomers and are therefore incorrect because the septum and the atria can be visualized practically from every view. The true superior rim (superior most part of the aortic rim) is best visualized with this view. We recommend that it should be renamed the "superior rim view," Although we have omitted the superior rim in our revised diagram, the superior rim view is seen best by ICE and can be separated from the aortic rim (Fig. 6).

The short-axis view can be obtained with the catheter in flexed and locked position. The entire catheter is rotated clockwise to bring the probe underneath the Ao (near the TV). This is followed by slight adjustments with the anterior-posterior and left-right knobs. The aortic and posterior rims can be obtained in this view. Unlike TEE, 

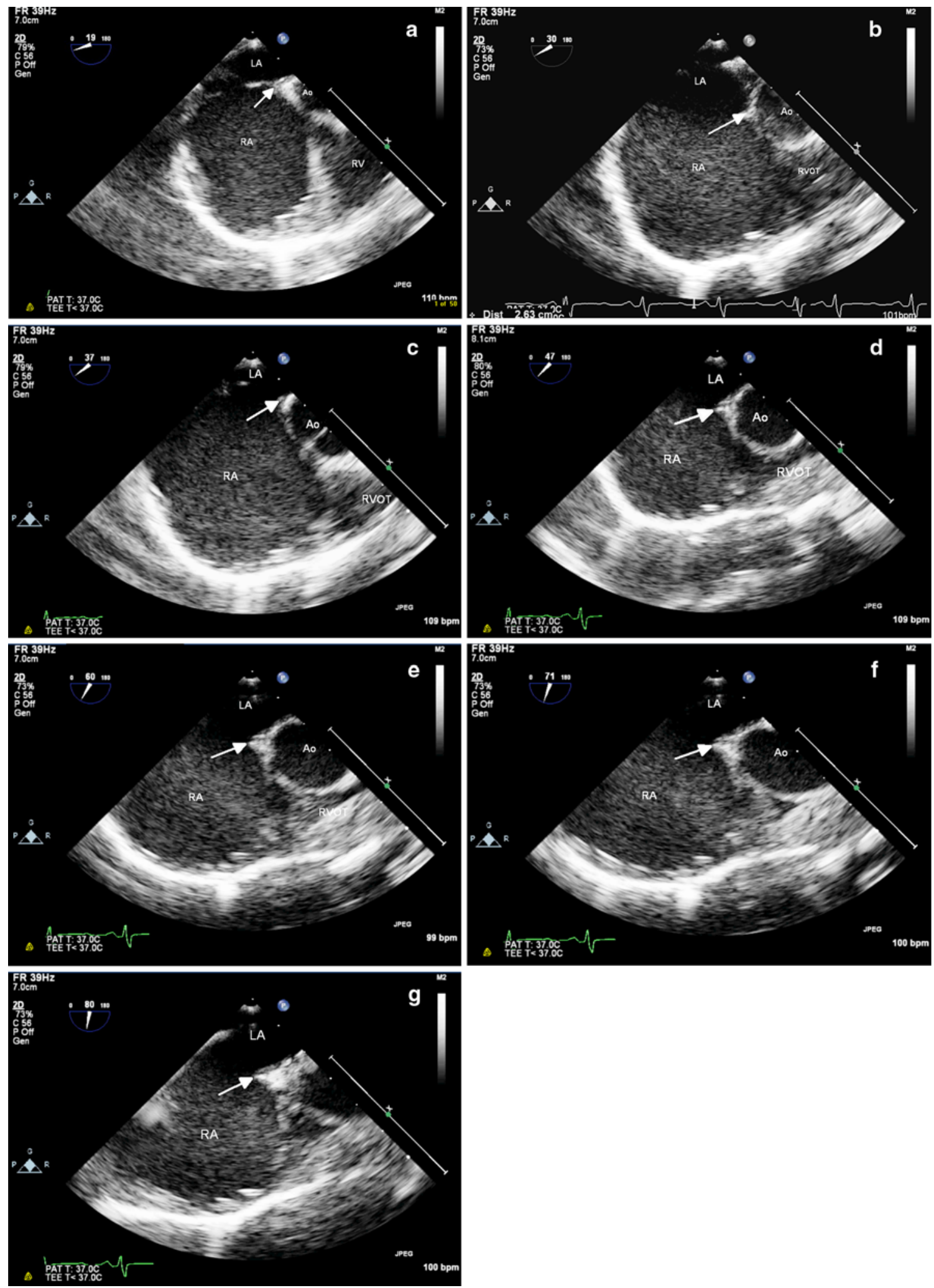

Fig. 4 Short-axis aortic sequential views. (a) Inferior portion of the aortic rim (arrow). (b-d) Deficient aortic rim $(<5 \mathrm{~mm})$ (arrow). (e-f) The aortic rim is sufficient (arrow). (g) Sufficient superior portion of

aortic rim (arrow). $L A$ left atrium; $R A$ right atrium; $A o$ aorta, $R V$ right ventricle, $R V O T$ right ventricular outflow tract 


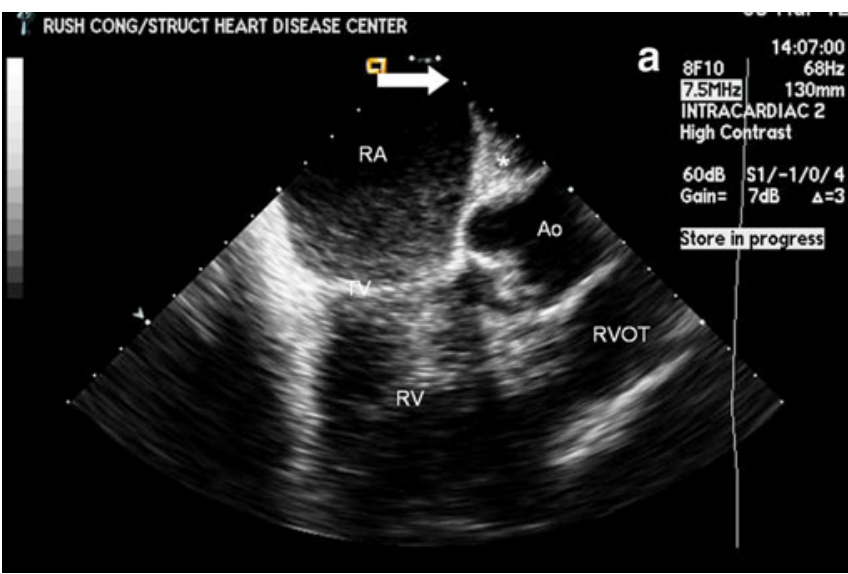

Fig. 5 (a) Neutral/home view showing the right atrium (RA), right ventricle (RV), right ventricular outflow tract (RVOT), Aorta (Ao), ASD (arrow), and the inferior portion of the aortic rim (*).

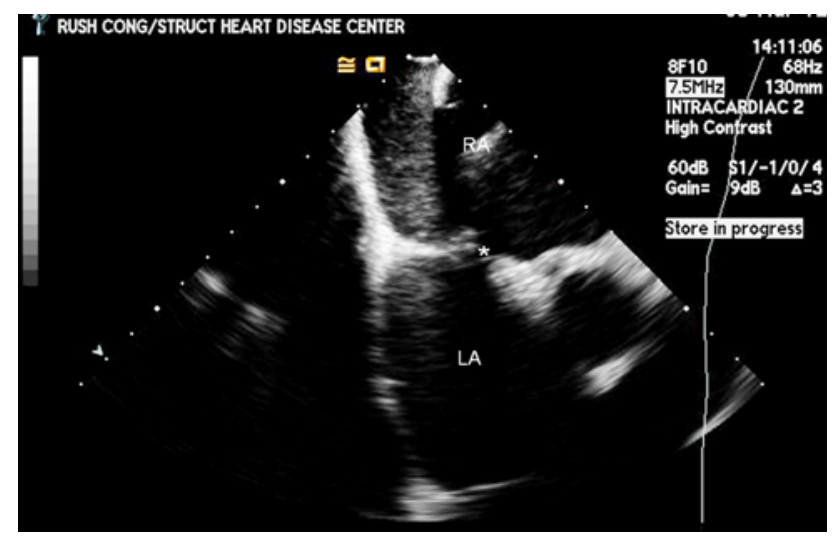

Fig. 6 Superior rim view in ICE shows the right atrium (RA), left atrium (LA) with the atrial septal defect (*). Occasionally, the right pulmonary venous return to the LA and the coronary sinus can be seen as well

there is no mechanism to angulate the ICE catheter to evaluate the aortic rim sequentially by ICE; however, mild adjustment and rotation (right-left) of the ICE catheter can

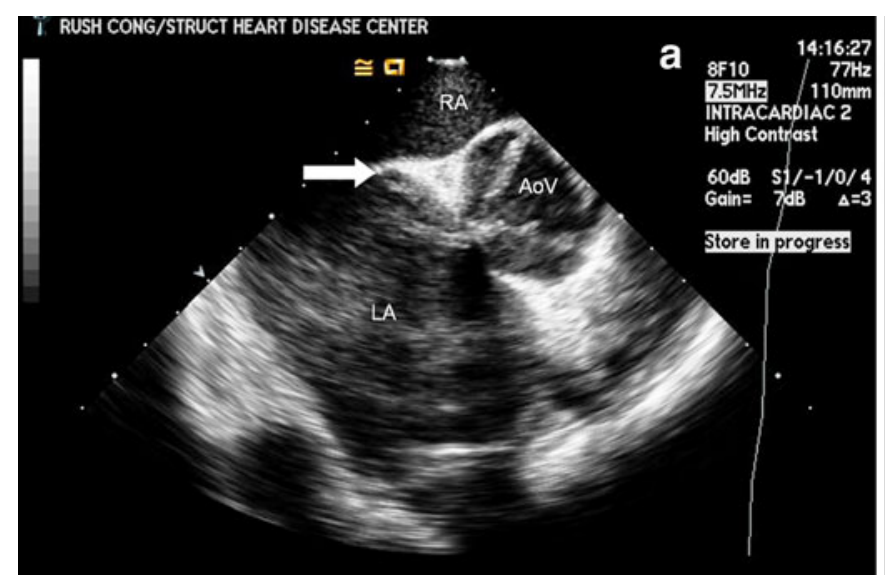

Fig. 7 (a) Low short-axis view in ICE shows the right atrium (RA), left atrium (LA), ASD, and the aortic valve (AoV) at the valvar level with three leaflets. The arrow points to the aortic rim. (b) High short-

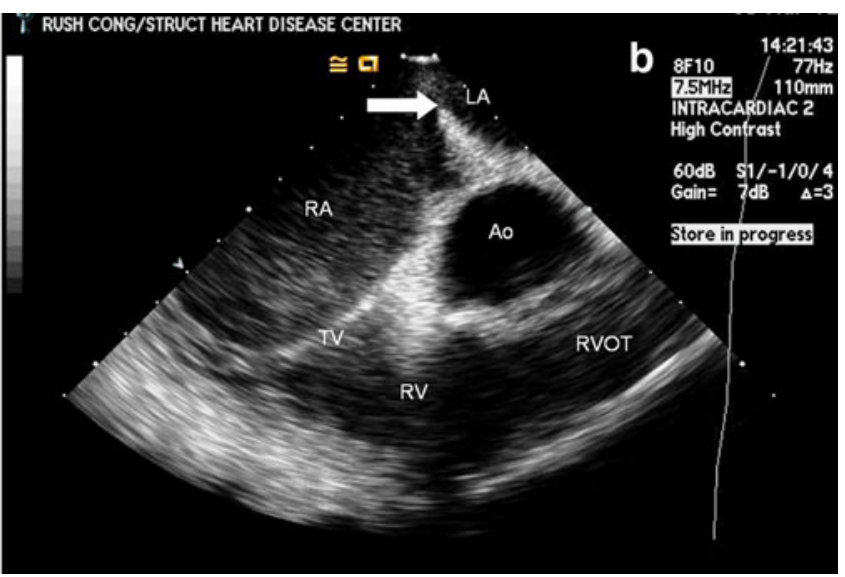

(b) Modified home view with portion of the left atrium (LA) and the inferior portion of the aortic rim (arrow)

help in visualizing the aortic rim close to the aortic sinus and above it (Fig. 7).

Hence, the home or neutral view, superior rim view, and short-axis view help in interrogating several parts of the aortic rim because it spans from the AV valve rim toward the start of the RUPV rim. Deficiency of the aortic rim at multiple levels will constitute a high-risk defect, (Fig. 6) for which ASD closure with an ASO is contraindicated. The bicaval view does not help in assessing any part of the aortic rim and hence has not been discussed.

\section{Conclusion}

Optimal intraprocedural imaging can help determine the adequacy of all rims of the secundum ASD, especially the aortic (anterior-superior) rim. In this report, we correlated the imaging views between ICE and TEE for appropriate

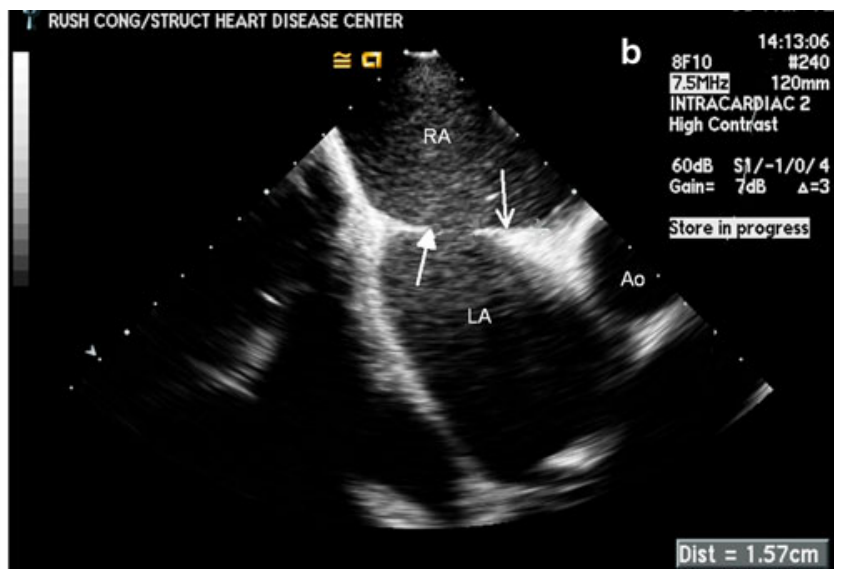

axis view in ICE demonstrates the right atrium (RA), left atrium (LA), ASD with the posterior rim (solid arrow), and the upper portion of aortic rim (open head arrow) 
assessment of the aortic rim of the secundum ASD. Deficiency of the aortic rim in multiple sequential views, either by ICE or TEE, would make ASD a high-risk defect contraindicating device closure. If, however, deficiency is noted in fewer than three consecutive and sequential views, device closure is not contraindicated. A clear understanding of the rims that surround the ASD is paramount to successful decision making and good outcomes.

\section{References}

1. Amin Z (2006) Transcatheter closure of secundum atrial septal defects. Catheter Cardiovasc Interv 68:778-787

2. Amin Z, Hijazi ZM, Bass JL, Cheatham JP, Hellenbrand WE, Kleinman CS (2004) Erosion of Amplatzer septal occluder device after closure of secundum atrial septal defects review of registry of complications and recommendations to minimize future risk. Catheter Cardiovasc Interv 63:496-502

3. Amin Z, Cao Q, Hijazi ZM (2009) Intracardiac echocardiography for structural heart defects: a review of ICE innovations, devices, and techniques. Cardiac Interv Today May:43-50

4. Amplatzer septal occluder and delivery system: instructions for use (2007-2009). Available at: http://www.amplatzer.com/por tals/aga/uploaded_docs/AMPLATZER_Septal_Occluder_Instruc tions_For_Use_United_States_Only_09_23_09.pdf. Accessed April $201 \overline{2}$

5. Bell-Cheddar Y, Amin Z (2011) Indications and evaluation for ASD closure: performing detailed hemodynamic evaluation of secundum atrial septal defects using current imaging modalities and closure devices. Card Interv Today September:1-6

6. Delaney JW, Li JS, Rhodes JF (2007) Major complications associated with transcatheter atrial septal occluder implantation: a review of the medical literature and the manufacturer and user facility device experience (MAUDE) database. Congenit Heart Dis 2:256-264

7. DiBardino D, McElhinney D, Kaza A, Mayer J Jr (2009) Analysis of the US food and drug administration manufacturer and user facility device experience database for adverse events involving Amplatzer septal occluder devices and comparison with the society of thoracic surgery congenital cardiac surgery database. J Thorac Cardiovasc Surg 137:1334-1341

8. El-Said HG, Moore JW (2009) Erosion by the Amplatzer septal occluder: experienced operator opinions at odds with manufacturer recommendations? Catheter Cardiovasc Interv 73:925-930

9. Kleinman CS (2005) Echocardiographic guidance of catheterbased treatments of atrial septal defect: transesophageal echocardiography remains the gold standard. Pediatr Cardiol 26: 128-134

10. Knepp MD, Rocchini AP, Lloyd TR, Aiyagari RM (2010) Longterm follow up of secundum atrial septal defect closure with the Amplatzer septal occluder. Congenit Heart Dis 5:32-37

11. Podnar T, Martanovic P, Gavora P et al (2001) Morphological variations of secundum-type atrial septal defects: feasibility for percutaneous closure using Amplatzer septal occluders. Catheter Cardiovasc Interv 53:386-391

12. Sadiq M, Kazmi T, Rehman AU, Latif F, Hyder N, Qureshi SA (2011) Device closure of atrial septal defect: medium-term outcome with special reference to complications. Cardiol Young $22: 1-8$ 